\title{
Theoretical transition probabilities, radiative lifetimes and Stark broadening parameters of singly ionized magnesium
}

\author{
C. Moreno-Díaz ${ }^{\circledR \star}$ and A. Alonso-Medina ${ }^{\odot}$ \\ Electricidad, Electrónica, Automática y Física Aplicada, E.T.S.I.D. Industrial Universidad Politécnica de Madrid, Ronda de Valencia 3, 28012 Madrid, Spain
}

Accepted 2019 September 26. Received 2019 September 26; in original form 2019 July 18

\begin{abstract}
The presence of spectral lines of singly ionized magnesium (Mg II) in stellar atmospheres has been reported in different stars. Recently, the low-resolution spectrum obtained from Supernova $2014 \mathrm{~J}$ in M82, in which Mg II absorption lines centred on $4400 \AA$ as well as $7600 \AA$ stand out, has been analysed. This is the motive for the atomic data calculations in this work, which are of much interest in the astrophysical area. In this article, ab initio relativistic Hartree-Fock calculations in an intermediate coupling formalism using Cowan's code allowed us to obtain the required transition probabilities to calculate the theoretical radiative lifetimes for excited $n \mathrm{~S}-, n \mathrm{P}-, n \mathrm{D}-$ and $n \mathrm{~F}-$ states of singly ionized magnesium. An asymptotic dependence of lifetime $\left(\tau_{n l}\right)$ on the effective principal quantum number $\left(n^{*}\right)$ has been determined. Also, the Griem semi-empirical approach was used to obtain the theoretical Stark parameters (width and shift) of spectral lines; these data are displayed for an electron density of $10^{17} \mathrm{~cm}^{-3}$ and temperatures $T=10-100\left(\times 10^{3} \mathrm{~K}\right)$. We have compared the results of lifetimes for 16 levels and Stark parameters for seven spectral lines with previously reported experiments available in the literature. Finally, we discuss the behaviour of the Stark parameters versus temperature for three relevant spectral lines (2802.70, 2797.99 and $7868.04 \AA$ ).
\end{abstract}

Key words: atomic data-atomic processes.

\section{INTRODUCTION}

Magnesium is one of the most abundant elements on Earth, with multiple applications. Magnesium is also present as an impurity in many industrial interest alloys, making it a good candidate for diagnosis of the properties of laser-produced plasma (LSP) from these alloys: see Moreno-Díaz (2017). LSP industrial application requires a parallel investigation of the formed plasma from a fundamental point of view: see, for example, the recent work by Moreno-Díaz et al. (2016) on a method applied to treated material in laser shock processing (LSP) processes.

Also, magnesium is an important chemical element for studying the history of $\alpha$-process nucleosynthesis. This element is observed relatively easily in the spectra of stars, even in metal-poor star atmospheres, and its overabundance in extremely metal-poor stars is one of the fundamental parameters of chemical evolution models for our Galaxy (Civiš et al. 2013). Spectral lines of singly ionized magnesium (Mg II) are of importance for the investigation, diagnostics and modelling of stellar atmospheres. In the solar spectrum, the triplet of lines $(2790.77,2797.93$ and $2797.99 \AA)$ is shown along with the resonance $h$ and $k$ lines (2802.70 and $2975.53 \AA$ ) of $\mathrm{Mg}$ II, mainly in absorption and in some cases also as lines of emission; see Morrill \& Korendyke (2008) and

^E-mail: cristina.mdiaz@upm.es
Pereira et al. (2015). Vallely et al. (2016) analysed a time series of optical spectra of Supernova $2014 \mathrm{~J}$ in M82 and at early times the spectral features are dominated by Si II, S II, Mg II and Ca II. Also, the Mg II triplet lines (4481.33, 4481.15 and $4481.13 \AA$ ) and (7898.04, 7877.05 and 7896.37 $\AA$ ) appeared as absorption lines. Also, this last triplet has been used by Lambert \& Warner (1968) and Vennes, Kawka \& Németh (2011) for the determination of the abundance of magnesium, first in the solar photosphere and also in the white dwarf GALEX J193156.8+011745. Therefore, we think that the calculation of transition probabilities, radiative lifetimes and Stark broadening parameters is necessary. For all these reasons, a theoretical study of the aforementioned atomic data of $\mathrm{Mg}$ II is presented in this work.

$\mathrm{Mg}$ II has ground configuration $1 \mathrm{~s}^{2} 2 \mathrm{~s}^{2} 2 \mathrm{p}^{6} 3 \mathrm{~s}^{1} \mathrm{~S}_{1 / 2}$ and excited states of type $1 \mathrm{~s}^{2} 2 \mathrm{~s}^{2} 2 \mathrm{p}^{6} \mathrm{nl}$. The energy levels and transition data can be found in Martin \& Zalubas (1980), Moore (1970) and Kaufman \& Martin (1991). Furthermore, Biétmot \& Brault (1986) measured the spectra of $\mathrm{Mg}$ I and $\mathrm{Mg}$ II produced by a hollow-cathode discharge observed by the Fourier transform spectroscopy technique between 1800 and $9000 \mathrm{~cm}^{-1}$. This observation has allowed an extension of the term systems of $\mathrm{Mg} \mathrm{I}$ and $\mathrm{Mg}$ II, as well as the values of the relative intensities of the observed spectral lines. This extension is collected in the National Institute of Standards and Technology (NIST) database (Kramida et al. 2018).

Theoretical works on transition probabilities can found in the bibliography, but there are no experimental ones. However, 
radiative lifetimes of $\mathrm{Mg}$ II have been measured by several authors. In chronological order, the lifetime of the $2 \mathrm{p}^{6} 3 \mathrm{p}^{2} \mathrm{P}^{0}{ }_{1 / 2}$ level was measured by Smith \& Gallagher (1966) using the Hanle-effect method with optical excitation. Berry, Bromander \& Buchta (1970) measured the lifetime levels $2 \mathrm{p}^{6} 4 \mathrm{~s}^{2} \mathrm{~S}_{1 / 2}, 2 \mathrm{p}^{6} 3 \mathrm{p}^{2} \mathrm{P}^{0}{ }_{1 / 2}, 2 \mathrm{p}^{6} 3 \mathrm{~d}^{2} \mathrm{D}_{3 / 2}$ and $2 \mathrm{p}^{6} \mathrm{f}^{2} \mathrm{~F}^{0} 5 / 2$ by mean of the beam-foil technique. Andersen et al. (1970), using the beam-foil excitation technique, measured the $2 \mathrm{p}^{6} 3 \mathrm{p}^{2} \mathrm{P}^{0}{ }_{1 / 2}$ level. The radiative lifetimes of the $3 \mathrm{p}^{2} \mathrm{P}^{0} \rightarrow$ $3 \mathrm{~s}^{2} \mathrm{~S}_{1 / 2}$ multiplets at 2795.53 and $2802.70 \AA$ were measured by Smith \& Lyszt (1971) using a phase-shift technique. The beam-foil technique has been used to measure the radiative lifetimes of the transitions $4 \mathrm{~d}^{2} \mathrm{D} \rightarrow 4 \mathrm{p}^{2} \mathrm{P}^{0}, 6 \mathrm{f}^{2} \mathrm{~F}^{0} \rightarrow 3 \mathrm{~d}^{2} \mathrm{D}, 6 \mathrm{f}^{2} \mathrm{~F}^{0} \rightarrow 4 \mathrm{~d}^{2} \mathrm{D}$ and $7 \mathrm{f}^{2} \mathrm{~F}^{0} \rightarrow 4 \mathrm{~d}^{2} \mathrm{D}$ by Andersen, Mølhave \& Sørensen (1972). With the same technique, Lundin et al. (1973) have determined radiative lifetimes of states $4 \mathrm{~s}^{2} \mathrm{~S}, 3 \mathrm{p}^{2} \mathrm{P}^{0}, 3 \mathrm{~d}^{2} \mathrm{D}, 4 \mathrm{p}^{2} \mathrm{P}^{0}$ and $4 \mathrm{f}^{2} \mathrm{~F}^{0}$. Lifetime measurements for 11 levels have been made by Liljeby et al. (1980), also using the beam-foil technique.

In the case of Stark parameters of Mg II (widths and shifts), there are also some experimental values in the bibliography. Stark width parameters from lines ( $3 \mathrm{~s}-3 \mathrm{p}$ transition, $2802.70 \AA$ and $3 \mathrm{~d}-4 \mathrm{f}$ transition, $4481.33 \AA$ ) have been measured in an argon plasma jet by Chapelle \& Sahal-Bréchot (1970). Using an electromagnetically driven shock tube and a rapid-scanning Fabry-Perot spectrometer, Jones et al. (1972) have measured Stark widths and shifts of resonance $h$ and $k$ lines (2802.70 and $2975.53 \AA$ ). The Stark widths and shifts of the 2795.53- and 2936.51- $\AA$ lines have been measured in an argon-welding type plasma by Fleurirer, Sahal-Bréchot \& Chapelle (1977). Goldbach et al. (1982) have measured resonance lines $h$ and $k$ again, but with wall-stabilized arc burning in argon with a small addition of $\mathrm{Mg}$ vapour.

Stark widths and shifts of six Mg II spectral lines very important in astrophysical situations (2790.77, 2795.53, 2797.99, 2802.70, 2928.63 and $2936.51 \AA$ ) have been measured by Djeniže et al. (2004) with a linear low-pressure arc as a plasma source. In the same way, Bukvić, Srećković \& Djeniže (2004) have measured resonance $h$ and $k$ spectral lines and values of the 2790.77- and 2797.99- $\AA$ lines in the $3 \mathrm{p}-3 \mathrm{~d}$ transition. Djeniže, Srećković \& Bukvić (2005) have again measured the Stark width and shift of the 4481.13- $\AA$ spectral line in the $3 \mathrm{~d}^{2} \mathrm{D}_{5 / 2}-4 \mathrm{f}^{2} \mathrm{~F}^{0} 7 / 2$ transition in nitrogen plasma generated in an optically thin linear, low-pressure, pulsed arc discharge. The Stark broadening parameter of the same line was also measured by Cvejić et al. (2013) with a laser-induced plasma.

In this article, we present theoretical values of transition probabilities for 320 spectral lines of $\mathrm{Mg}$ II that are shown in the NIST database and arise from configurations $2 \mathrm{p}^{6} n \mathrm{~s}(n=4-10), 2 \mathrm{p}^{6} n \mathrm{p}$ $(n=3-9), 2 \mathrm{p}^{6} n \mathrm{~d}(n=3-8)$ and $2 \mathrm{p}^{6} n \mathrm{f}(n=4-10)$. Lifetimes of 47 levels are also calculated and compared with experimental values available in the bibliography. These calculations have been made with ab initio relativistic Hartree-Fock (HFR) in an intermediate coupling (IC) formalism using Cowan's code. In Section 2, the method and the theoretical calculations are described. Following this, Section 3 provides the results and a discussion of transition probabilities, radiative lifetimes and Stark broadening parameters, including an analysis of the different regularities of these last.

\section{METHOD OF CALCULATION}

In this work, HFR calculations in an IC scheme have been realized. We have used a similar procedure to that described in previous works, such as Alonso \& Colón (2013), Moreno-Díaz, AlonsoMedina \& Colón (2014), Alonso-Medina, Colón \& Moreno-Díaz (2015) and Moreno-Díaz (2017). Wave functions and transition probabilities were obtained by the standard method of least-squares fitting of experimental energy levels, presented by Martin and Zalubas (1970), Moore (1971) and Kaufman \& Martin (1991), by means of Cowan's computer code (Cowan 1981). For the HFR calculations, the Cowan computer code provides not only the radial parts, for determination of transition probabilities, but also an initial estimation of the parameters for the IC fittings. Our set consists of 26 configurations, $2 \mathrm{p}^{6} n \mathrm{~s}(n=3-10), 2 \mathrm{p}^{6} n \mathrm{~d}(n=3-$ $9), 2 \mathrm{p}^{6} n \mathrm{~g}(n=5-11)$ and $2 \mathrm{p}^{6} n \mathrm{i}(n=7-10)$, of even parity, and 20 configurations of odd parity, $2 \mathrm{p}^{6} n \mathrm{p}(n=3-9), 2 \mathrm{p}^{6} n \mathrm{f}(n=4-$ $10)$ and $2 \mathrm{p}^{6} n \mathrm{~h}(n=6-11)$ of $\mathrm{Mg}$ II. Since magnesium is a light element, core polarization effects have not been introduced. The values obtained with the HFR ab initio calculations were used as a starting point in the setting of energy levels. Thus, we obtained the LS composition of each level and the degree of configuration mix, considering their interactions and the experimental energy levels. Fig. S1 (available online) displays a partial Gotrian energy-level scheme of Mg II, showing the adjusted levels and spectral lines, previously mentioned, of greater astrophysical interest.

Because of the high number of parameters to adjust (which exceeds of the number of experimental levels), we have excluded a certain number of parameters from the adjustment process. The scaling factors of the Slater parameters $\left(F^{k}\right.$ and $\left.G^{k}\right)$ and configuration interaction integrals $\left(R^{k}\right)$, not optimized in the least-squares fitting, were chosen to equal 0.85, as suggested by Cowan (1981). For the spin-orbit integrals $\zeta_{n l}$, characterized by small numerical values and no adjustment in the fitting procedures, we have used the HFR ab initio values without scaling. The values found for the different parameters involved in the IC calculations, compared with HFR (used as a start in the fitting process), are shown in Table S1 (available online). The wave functions obtained in Cowan's code were used in the calculations to obtain the matrix elements required.

The transition probabilities are obtained from the matrix elements by using the standard expression of Martin \& Wiese (1996):

$A_{n l}=\frac{2 \pi e^{2}}{m_{\mathrm{e}} c \varepsilon_{0} \lambda^{2}} \frac{g_{l}}{g_{n}} f_{l n}=\frac{16 \pi^{3}}{3 h \varepsilon_{0} \lambda^{3} g_{n}}\left|\left\langle P_{n}|\boldsymbol{r}| P_{l}\right\rangle\right|^{2}$,

where $A_{n l}$ and $f_{l n}$ are the transition probability and the oscillator strength, respectively, $e$ and $m_{\mathrm{e}}$ are the electron charge and electron mass, $\lambda$ is the transition wavelength, $c$ is the light speed, $h$ is the Planck constant, $g_{l}$ and $g_{n}$ are the statistical weights and $\left\langle P_{n}|\boldsymbol{r}| P_{l}\right\rangle$ is the calculated matrix element including the modification pointed out above. Once all the probabilities of transition are known that come from a level, also the lifetime of that level are known.Then the only possibility to compare the results of probabilities is with other theoretical works. In this article, this comparation is not realized because discrepancies can be present, in many cases due to the theoretical approach of each author. However, the calculated radiative lifetimes have been compared with the experimental values of other authors. In Tables S2-S5 (available online), the theoretical transition probabilities and radiative lifetimes of some levels of $\mathrm{Mg}$ II are shown. The ratio between the theoretical lifetimes of this study and the previously measured experimental lifetimes can be seen in Fig. S2 (available online).

As we have already indicated, information on the lifetimes is necessary for estimating use of the Rydberg states for practice applications. Theoretical investigations of the optical properties of Rydberg states in $\mathrm{Mg}$ II are of current interest. In this work we obtained approximations of lifetimes for excited $n \mathrm{~S}-, n \mathrm{P}-, n \mathrm{D}-$ and $n \mathrm{~F}-$ states, with the equation

$\tau_{n l}=\tau_{0}\left(n^{*}\right)^{\alpha}$, 
where $n^{*}$ is the effective principal quantum number and $\tau_{0}$ and $\alpha$ are two constants, applicable to states with principal quantum number in the range $n=3-10$. This expression is used by several authors: see as an example Rykova \& Verolainen (1994) and Glukhov et al. (2013). The constant $\tau_{0}$ for different series of states, as shown in Table S6 (available online), range from $\tau_{0}=0.05 n \mathrm{~s}$ of $n \mathrm{~F}-$ states to $\tau_{0}=0.62 n \mathrm{~s}$ of $n \mathrm{P}-$ states, On the other hand, the value of the exponent is around $\alpha=3$, with a deviation in the results of less than \pm 0.5 . Fig. S3 (available online) shows the representation of the lifetime of the levels versus the effective principal quantum number of each level corresponding to different series $\left(n \mathrm{~s}^{2} \mathrm{~S}_{1 / 2}, n \mathrm{p}^{2} \mathrm{P}^{0}{ }_{1 / 2}\right.$, $\left.n \mathrm{~d}^{2} \mathrm{D}_{3 / 2}, n \mathrm{f}^{2} \mathrm{~F}^{0}{ }_{5 / 2}\right)$. The values of the half-lives measured by Smith \& Gallagher (1966), Andersen et al. (1970), Berry et al. (1970), Smith \& Lyszt (1971), Lundin et al. (1973), Liljeby et al. (1980) and Andersen et al. (1970) can be seen together. It is verified that said half-lives follow expression (2) and the corresponding alpha values can be observed for each graph. These values confirm the asymptotic nature of the dependence of $\tau_{n l} \propto\left(n^{*}\right)^{3}$, indicating that, for the studied levels, the model used in this work is applicable with good precision.

On the other hand, previously determined matrix elements have also been used in the calculation of Stark broadening parameters from the semi-empirical approach suggested by Griem (1968), using his semi-empirical formulae (23) and (35). This calculation procedure is similar to those presented in recent publications by the authors Colón, Moreno-Díaz \& Alonso-Medina (2013), AlonsoMedina \& Colón (2014), Moreno-Díaz et al. (2014) and recently Moreno-Díaz (2017). This approach was obtained taking into account Baranger's original formulation (Baranger 1958) and the use of an effective Gaunt factor, proposed by Seaton (1962) and Van Regemorter (1962).

In Tables S7-S10 (available online), the theoretical Stark width and shift parameters for some levels of $\mathrm{Mg}$ II are shown. In Fig. S4 (available online), we compared the dependence on the Stark parameters for several spectral lines of $\mathrm{Mg}$ II with the electron temperature.

\section{RESULTS AND DISCUSSION}

\subsection{Transition probabilities and radiative lifetimes}

In Tables S2-S5 (available online), we present the results obtained in this work for transition probabilities of 320 spectral lines in the wavelength range 870-180000 $\AA$ and lifetimes of 47 levels of Mg II. Of these last, 16 have been compared with experimental values available in the literature. In the first column, we present the transition (the upper and lower level). The second one displays the wavelength in $\AA$ (see Martin \& Zalubas 1980). In the third column we show the relative intensity $I_{\text {rel }}$ (the intensities of lines referred to the intensities of $\mathrm{Mg}$ I lines at 2150.353 and 5843.407 $\mathrm{cm}^{-1}$, Biétmont \& Brault 1986). The remaining columns present our values: the fourth column shows the transition probabilities and in the fifth our radiative lifetimes are compared with the experimental values of other authors (Smith \& Gallagher 1966; Andersen et al. 1970; Berry et al. 1970; Smith \& Lyszt 1971; Lundin et al. 1973; Liljeby et al. 1980; Andersen et al 1970).

In order to facilitate the comparison, in Fig. S2 (available online) we present the ratio between experimental values of radiative lifetimes and the theoretical values obtained in this work for several levels of $\mathrm{Mg}$ II ( $\tau_{\text {exp }} / \tau_{\text {this }}$ work) versus the energy of the levels. As can be seen, our values are in good agreement with the experimental ones, with the exception of some levels. In any case, the deviations are less than 10 per cent. Andersen et al. (1970) and Lundin et al. (1973) found that the radiative lifetime of the doublet $(2802.70$ and $2795.53 \AA$ ) was difficult to solve. They had to study this lifetime using line $2802.70 \AA$, because line $2795.53 \AA$ is mixed with line $2790.77 \AA$ of the transition $3 \mathrm{p}^{2} \mathrm{P}^{\mathrm{o}}{ }_{1 / 2}-3 \mathrm{~d}{ }^{2} \mathrm{D}_{3 / 2}$. They found an appreciable cascading in this transition that complicates the interpretation of the obtained lifetime. Also, in $4 \mathrm{p}^{2} \mathrm{P}^{\mathrm{o}}-4 \mathrm{~d}$ ${ }^{2} \mathrm{D}$ and $3 \mathrm{~d}{ }^{2} \mathrm{D} \_6 \mathrm{f}{ }^{2} \mathrm{~F}^{\mathrm{o}}$ transitions, Andersen et al. (1972) found cascading.

Fig. S3 (available online) shows the representation of the lifetimes of the levels $n \mathrm{~s}^{2} \mathrm{~S}_{1 / 2}, n \mathrm{p}^{2} \mathrm{P}^{\mathrm{o}}{ }_{1 / 2}, n \mathrm{~d}^{2} \mathrm{D}_{3 / 2}$ and $n \mathrm{f}^{2} \mathrm{~F}^{\mathrm{o}}{ }_{5 / 2}$ studied against the effective principal quantum number of each level corresponding to each series. As can be seen, the mentioned lifetimes follow expression (2). In addition, in each graph the values of $\alpha$ can be appreciated and we corroborate that these values are close to 3 . Values of constants $\tau_{\mathrm{o}}$ and $\alpha$ for all series studied are presented in Table S6 (available online).

In conclusion, regarding these calculations, we can say that the transition probabilities as well as the lifetimes of their different states can be calculated with reasonable safety using ab initio HFR. For this reason, we think that these values are a good basis for the calculations of the of Stark broadening parameters presented below.

\subsection{Stark width FWHM and Stark line shift}

The results for the Stark line width and line shift at an electron density of $10^{17} \mathrm{~cm}^{-3}$ and several temperatures $\mathrm{T}=10-100\left(\times 10^{3} \mathrm{~K}\right)$ are displayed in Tables S7-S10 (available online). The first three columns denote the corresponding transition array, the multiplet and the wavelengths (in $\AA$ : Martin \& Zalubas 1980) for each transition studied. The temperatures (in K) are shown in the fourth column. Theoretical Stark broadening line widths (in pm) are displayed in the fifth column and, where appropriate, the experimental values from the literature. The final column displays the theoretical Stark line shift (a positive shift is red) and in the same way the experimental values of other authors: see Cappelle \& Sahal-Bréchot (1970), Jones et al. (1972), Fleurier et al. (1977), Goldbach et al. (1982), Bukvić et al. (2004), Djeniže et al. (2004), Djeniže et al. (2005) and Cvejić et al. (2013). Our Stark full width at half-maximum (FWHM) values are in good agreement with the experimental ones, with some exceptions. For example, in the values of the $\mathrm{Mg}$ II resonance doublet measured by Goldbach et al. (1982), the width was found lower by a factor of 2 than all previous experimental values, as mentioned by these authors.

The dependence of Stark parameters on electron temperature is shown in Fig. S4 (available online). In this figure we represent the values of calculated Stark width FWHM $\omega(\mathrm{pm})$ and Stark line shift $d(\mathrm{pm})$ versus temperature for three lines.

The lines 2802.70 and $2797.99 \AA$ are presented in comparation with the experimental values of other authors. These lines could be used in the diagnosis of plasmas. We show the line $7898.04 \AA$, which in addition to being used in plasma diagnosis also has great astrophysical interest and belongs to a multiplet, arising from levels with high energies of the order of $93000 \mathrm{~cm}^{-1}$. As expected, the trends found coincide with those anticipated theoretically.

To conclude, using Cowan's code we have obtained the matrix elements necessary for the calculation of the Stark broadening parameters of $\mathrm{Mg}$ II spectral lines. We know that there are different ways to perform these calculations, but we have used Griem's semiempirical method. This method has given us good results within the margins of experimental error. 


\section{ACKNOWLEDGEMENTS}

This work was financially supported by the Spanish Ministry of Science and Technology (DGI project MAT-2015-63974-C4-2-R).

\section{REFERENCES}

Alonso-Medina A., Colón C., 2013, MNRAS, 431, 2703

Alonso-Medina A., Colón C., 2014, MNRAS, 445, 1567

Alonso-Medina A., Colón C., Moreno-Díaz C., 2015, Atomic Data and Nuclear Data Tables, 102, 64

Andersen T., Desesquelles J., Jessen K. A., Sørensen G., 1970, J. Quant. Spectrosc. Radiative Transfer, 10, 1143

Andersen T., Mølhave L., Sørensen G., 1972, ApJ, 178, 577

Baranger M. 1958, Phys. Rev, 112, 855

Berry H. G., Bromander J., Buchta R., 1970, Phys. Scripta, 1, 181

Biémont E., Brault J. W.,1986, Phys. Scripta, 34, 751

Bukvić S., Srećković A., Djeniže S., 2004, New Astron., 9, 629

Chapelle J., Sahal-Bréchot S., 1970, A\&A, 6, 415

Civiš S., Ferus M., Chernov V. E., Zanozina E. M., 2013, A\&A, 554, A24

Colón C., Moreno-Díaz C., Alonso-Medina A., 2013, MNRAS, 431, 2703

Cowan R. D., 1981, The Theory of Atomic Structure and Spectra. University of California Press, Berkeley USA

Cvejić M., Gavrilović M. R., Jovićević S., Konjević N., 2013. Spectrochim. Acta Part B, 85, 20

Djeniže S., Bukvić S., Srećković A., Platiš M., 2004, A\&A, 424, 561

Djeniže S., Srećković A., Bukvić S., 2005, Japanese J.Applied Phys., 44, 1450

Fleurirer C., Sahal-Bréchot S., Chapelle J., 1977, J. Quant. Spectrosc. Radiative Transfer, 17, 595

Glukhov I. L, Nikitina E. A., Ovsiannikov V. D., 2013, Optics and Spectroscopy, 115, 12

Goldbach C., Nollez G., Plomdeur P., Zimmermann J. P., 1982, Phys. Rev. A, 25, 2596

Griem H. R., 1968, Phys. Rev., 165, 258

Jones W. W., Sanchez A., Greig J. R., Griem H. R., 1972, Phys. Rev. A, 5, 2318

Kaufman V., Martin M. C., 1991, J. Chem. Ref. Data, 20, 97

Kramida A., Ralchenko Yu., Reader J., NIST ASD Team., 2018, NIST Atomic Spectra Database (see. 5.6.1). National Institute of Standards and Technology, Gaithersburg, MD, (accessed 2019, July 10)

Lambert B., Warner B., 1968, MNRAS, 140, 147

Liljeby L., Lindgårg A., Mannervik S., Veje E., Jelenković B., 1980. Phys. Scripta, 21, 805

Lundin L., Engman B., Hilke J., Martinson I., 1973. Phys. Scripta, 8, 274

Martin W. C., Wiese W. L., 1996, Atomic, Molecular and Optical Physics Handbook, Atomic Spectroscopy. AIP Press, Woodbury, NY (Chapter 10)

Martin W. C., Zalubas R., 1980, J. Phys. Chem. Ref. Data, 9, 15

Moore C. E., 1971, Atomic Energy Levels, vol. 1, Nat. Stand. Ref. Data Ser., Nat. Bur. Satand 35, U.S.A, p. 359

Moreno-Díaz C., 2017, PhD thesis, Univ. Politécnica de Madrid

Moreno-Díaz C., Alonso-Medina A., Colón C., 2014, Phys. Scripta, 89, 115401

Moreno-Díaz C., Alonso-Medina A., Colón C., Porro J. A., Ocaña J. L., 2016, J. Mater. Process.Tech. 232, 9

Morrill J. S., Korendyke C. M., 2008, ApJ, 687, 646

Pereira T. M. D., Carlsson M., De Pontieu B., Hansteen V., 2015, ApJ, 806, 14

Rikova, O, Verolainen Y, 1994, Optics and Spectroscopy, 76, 23

Seaton M. J., 1962, in Bates D. R., eds, Atomic and Molecular Processes. New York Academic, New York

Smith W. H., Lyszt H. S., 1971, J. Opt. Soc. Am., 61, 938

Smith W. W., Gallagher A., 1966. Phys. Rev., 145, 26

Vallely P. et al., 2016, MNRAS, 460, 1614

Van Regemorter H., 1962, ApJ, 136, 906

Vennes S., Kawka A., Németh P., 2011, MNRAS, 413, 2545

\section{SUPPORTING INFORMATION}

Supplementary data are available at MNRAS online.

Figure S1. Partial Gotrian diagram of Mg II energy levels (Martin \& Zalubas 1980), showing several spectral lines of great astrophysical interest.

Figure S2. Ratio between experimental values of radiative lifetimes and the theoretical values obtained in this work for several levels of $\operatorname{Mg}$ II ( $\left.\tau_{\text {exp }} / \tau_{\text {this work }}\right)$ versus the energy of the levels.

Figure S3. Experimental lifetimes of the $1 \mathrm{~s}^{2} 2 \mathrm{~s}^{2} \mathrm{p}^{6}\left(n \mathrm{~s}{ }^{2} \mathrm{~S}_{1 / 2}, n \mathrm{p}\right.$ ${ }^{2} \mathrm{P}^{0}{ }_{1 / 2}$, nd ${ }^{2} \mathrm{D}_{3 / 2}, n \mathrm{f}^{2} \mathrm{~F}^{0}{ }_{5 / 2}$ ) series in $\mathrm{Mg}$ II as a function of the effective principal quantum number $n^{*}$ : black filled circles - our work, red open circles - Liljeby et al. (1980), green diamonds Berry et al. (1970), blue upwards-pointing triangles - Andersen et al. (1970), magenta downwards-pointing triangles - Lundin et al. (1973), orange left-pointing triangles - Smith \& Gallagher (1966), light green right-pointing triangles - Smith \& Lyszt (1971), purple x symbols - Andersen et al. (1970).

Figure S4. Calculated Stark width FWHM $(\omega(\mathrm{pm}))$ and shift $(d$ $(\mathrm{pm})$ ) at an electron density of $1017 \mathrm{~cm}^{-3}$ versus temperature and experimental values from other authors, for 2802.70-, 2797.99- and 7898.04- ̊ spectral lines of Mg II.

Table S1. Parameters resulting from the least-squares fitting of the intermediate coupling calculations.

Table S2. $\mathrm{Mg}$ II $1 \mathrm{~s}^{2} 2 \mathrm{~s}^{2} 2 \mathrm{p}^{6} n s(n=4-10)$ relative intensity $I_{\text {rel }}$ (the intensities of the lines are referred to the intensities of $\mathrm{Mg}$ I lines at 2150.353 and $5843.407 \mathrm{~cm}^{-1}$ : Biétmont \& Brault 1986), transition probabilities $A_{i j}$ (in $10^{7} \mathrm{~s}^{-1}$ ) and radiative lifetimes $\tau$ (in $\mathrm{ns}$ ).

Table S3. $\mathrm{Mg}$ II $1 \mathrm{~s}^{2} 2 \mathrm{~s}^{2} 2 \mathrm{p}^{6} n p(n=3-9)$ relative intensity $I_{\text {rel }}$ (the intensities of the lines are referred to the intensities of $\mathrm{Mg}$ I lines at 2150.353 and $5843.407 \mathrm{~cm}^{-1}$ : Biétmont \& Brault 1986), transition probabilities $A_{i j}$ (in $10^{7} \mathrm{~s}^{-1}$ ) and radiative lifetimes $\tau$ (in ns).

Table S4. Mg II $1 \mathrm{~s}^{2} 2 \mathrm{~s}^{2} 2 \mathrm{p}^{6}$ nd $(n=3-8)$ relative intensity $I_{\text {rel }}$ (the intensities of the lines are referred to the intensities of $\mathrm{Mg}$ I lines at 2150.353 and $5843.407 \mathrm{~cm}^{-1}$ : Biétmont \& Brault 1986), transition probabilities $A_{i j}$ (in $10^{7} \mathrm{~s}^{-1}$ ) and radiative lifetimes $\tau$ (in ns).

Table S5. Mg II $1 \mathrm{~s}^{2} 2 \mathrm{~s}^{2} 2 \mathrm{p}^{6} n f(n=4-10)$ relative intensity $I_{\text {rel }}$ (the intensities of the lines are referred to the intensities of $\mathrm{Mg}$ I lines at 2150.353 and $5843.407 \mathrm{~cm}^{-1}$ : Biétmont \& Brault 1986), transition probabilities $A_{i j}$ (in $10^{7} \mathrm{~s}^{-1}$ ) and radiative lifetimes $\tau$ (in ns).

Table S6. Values of constants $\tau_{\mathrm{o}}$ and $\alpha$ for different series of excited states of $\mathrm{Mg}$ II.

Table S7. Mg II $1 \mathrm{~s}^{2} 2 \mathrm{~s}^{2} 2 \mathrm{p}^{6} n \mathrm{~s}(n=4-6)$ line widths (FWHM) $\omega$ (pm) and shifts $d(\mathrm{pm})$ normalized to $N_{\mathrm{e}}=10^{17} \mathrm{~cm}^{-3}$.

Table S8. Mg II $1 \mathrm{~s}^{2} 2 \mathrm{~s}^{2} 2 \mathrm{p}^{6} n \mathrm{p}(n=3-5)$ line widths (FWHM) $\omega$ (pm) and shifts $d(\mathrm{pm})$ normalized to $N_{\mathrm{e}}=10^{17} \mathrm{~cm}^{-3}$.

Table S9. Mg II $1 \mathrm{~s}^{2} 2 \mathrm{~s}^{2} 2 \mathrm{p}^{6} n \mathrm{~d}(n=3-5)$ line widths (FWHM) $\omega$ (pm) and shifts $d(\mathrm{pm})$ normalized to $N_{\mathrm{e}}=10^{17} \mathrm{~cm}^{-3}$.

Table S10. Mg II $1 \mathrm{~s}^{2} 2 \mathrm{~s}^{2} 2 \mathrm{p}^{6} 4 \mathrm{f}$ line widths (FWHM) $\omega(\mathrm{pm})$ and shifts $d(\mathrm{pm})$ normalized to $N_{\mathrm{e}}=10^{17} \mathrm{~cm}^{-3}$.

Please note: Oxford University Press is not responsible for the content or functionality of any supporting materials supplied by the authors. Any queries (other than missing material) should be directed to the corresponding author for the article.

This paper has been typeset from a Microsoft Word file prepared by the author. 